\title{
Smartphone-Read Phage Lateral Flow Assay for Point-of-Care Detection of SARS-CoV-2 Infection
}

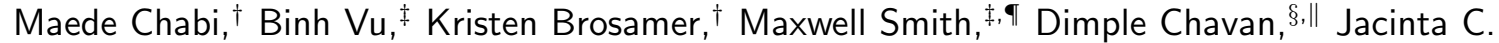 \\ Conrad, ${ }^{*, \ddagger}$ Richard C. Willson, ${ }^{*, \ddagger, \S, \dagger}$ and Katerina Kourentzi ${ }^{*, \ddagger}$ \\ $\dagger$ Department of Biomedical Engineering, University of Houston, Houston, TX 77204-5060 \\ $\ddagger$ Department of Chemical and Biomolecular Engineering, University of Houston, Houston, TX 77204-4004 \\ ๑ Current address: Merck, Kenilworth, NJ 07033 \\ $\S$ Department of Biology and Biochemistry, University of Houston, Houston, TX 77204-5001 \\ ||Current address: Purification Process Sciences, AstraZeneca, Gaithersburg, MD 20878 \\ Received February 3, 2022; E-mail: jcconrad@uh.edu; willson@uh.edu; edkourentzi@uh.edu
}

\begin{abstract}
:
The COVID-19 pandemic has highlighted the urgent need for sensitive, affordable, and widely-accessible testing at the point-of-care. Here we report the development of a sensitive chemiluminescence-based smartphone-readable lateral flow assay for the detection of SARS-CoV-2 nucleoprotein using M13 phage conjugated with antibodies and HRP enzymes as LFA reporter particles. We screened 84 anti-nucleoprotein monoclonal antibody pairs in phage LFA and identified an antibody pair that gave an LoD of $25 \mathrm{pg} \mathrm{mL}^{-1}$ nucleoprotein in nasal swab extract using a FluorChem gel documentation system and $100 \mathrm{pg} \mathrm{mL}^{-1}$ when the test was imaged and analyzed by an in-house-developed smartphone reader. The smartphone-read LFA signals for positive clinical samples tested $(N=15$, with known $\mathrm{Ct}$ ) were statistically different $(p<0.001)$ from negative clinical samples tested $(N=12)$. The translation-ready phage LFA technology combined with smartphone chemiluminescence imaging can enable the timely development of ultrasensitive, affordable point-of-care testing platforms for SARS-CoV-2 and beyond.
\end{abstract}

\section{Introduction}

Coronavirus disease 2019 (COVID-19), caused by severe acute respiratory syndrome coronavirus 2 (SARS-CoV-2), has become a major global health crisis since early 2020 . Rapid SARS-CoV-2 diagnostic antigen tests are a point-ofcare alternative to gold standard RT-PCR for frequent and affordable testing, ${ }^{1-3}$ used to identify and isolate infected people as early as possible to control the transmission. ${ }^{4}$ Rapid antigen tests most commonly target the viral nucleoprotein ( $\mathrm{N}$ protein $)^{5-12}$ and can test for current infection at the point-of-care. ${ }^{13,14}$

The nucleoprotein is a highly conserved and abundantly expressed viral structural protein in SARS-CoV-2 and becomes accessible after a simple detergent-based sample treatment. Thus, it is the preferred diagnostic target (over the mutation-prone surface trimer spike protein). Indeed, the majority of the SARS-CoV-2 antigen tests under FDA Emergency Use Authorization target the nucleoprotein. ${ }^{15}$

Lateral flow immunoassays (LFAs) are the preferred format for point-of-care antigen-detection diagnostics. When they have sufficient analytical sensitivity, they can be used in a wide array of diagnostic applications including screening for respiratory infections such as flu, ${ }^{16}$ respiratory syncytial virus (RSV) infection, ${ }^{17}$ and most recently COVID-19. ${ }^{15}$ LFAs are based on capillary wicking of a liquid sample along a nitrocellulose membrane bearing immobilized (capture) antibodies on test and control lines. As the sample wicks through the membrane, it contacts reporter particles decorated with antibodies to the target. The particle-antibodytarget complexes are captured by the anti-target antibodies immobilized on the test line, and particles are captured independent of the target on the control line, resulting in the lines characteristic of a positive LFA.

While antibodies play a key role in immunoassay performance, the analytical sensitivity of LFA is also determined by the detectability of the reporter particles. ${ }^{18}$ Conventional gold and latex colorimetric reporters give limited analytical sensitivity ${ }^{19}$ albeit without complex readouts. Higher-sensitivity reporters include organic fluors, ${ }^{20}$ europium chelates, ${ }^{21,22}$ quantum dots, ${ }^{10,11,23-25}$ up-converting nanoparticles, ${ }^{26}$ noble-metal catalytic nanoparticles, ${ }^{27}$ and paramagnetic nanoparticles. ${ }^{28,29}$ These reporters can greatly enhance LFA sensitivity but typically require costly and/or complex readers. ${ }^{30-32}$ Achieving high analytical sensitivity without sacrificing the simplicity, manufacturability, and low cost of LFA remains an open challenge.

We previously introduced filamentous M13 bacteriophage particles as LFA reporters combining enhanced detectability and capturability. ${ }^{33-38}$ The M13 phage reporters carry multiple recognition elements, e.g. antibodies or aptamers, and multiple copies of reporter enzymes or fluors for signal amplification. These phage reporters exhibited extremely low limits of detection in model systems or using sophisticated fluorescence instrumentation in mechanistic investigations of LFAs, ${ }^{33-38}$ but were not tested on real samples at the pointof-care.

In this study we show that phage LFAs employing chemiluminescent reporters and read by a smartphone can reliably detect SARS-CoV-2 in clinical samples. Through extensive screening of antibodies and conjugation chemistries, we demonstrate the ultrasensitive detection of SARS-CoV-2 nucleoprotein in nasal swab extracts using an off-the-shelf smartphone (fitted with a $\$ 1.203-\mathrm{D}$ printed accessory) and an in-house developed iOS app for imaging and analysis. 


\section{Materials and Methods}

\subsection{Materials}

Hydroxylamine hydrochloride (99\%), sodium meta periodate $(\geq 99 \%)$, IGEPAL CA-630, bovine serum albumin (A7906, $\geq 98 \%$ ), polyvinylpyrrolidone (PVP-40, average MW 40,000), and sodium acetate (99\%) were purchased from Sigma Aldrich and used without modification or purification. Sodium carbonate was from Millipore Sigma. Sodium cyanoborohydride was purchased from CHEMIMPEX and used without modification. Phosphate Buffered Saline (PBS) tablets were from TaKaRa. Enhanced Chemiluminescence (ECL) Ultra substrate (acridan-based; TMA6) was purchased from Lumigen and 1-Step Ultra TMBELISA Substrate Solution was from Thermo Scientific.

\subsection{Nasal swab samples}

For spiking studies, fresh presumed negative anterior nasal swab specimens were self-collected by adult lab members under a University of Houston IRB-approved study (UH STUDY00002547) using Puritan foam tip swabs. Each swab was extracted with $1 \mathrm{~mL}$ of LFA extraction buffer (1X PBS (pH 7.4), $10 \mathrm{mg} \mathrm{mL}^{-1} \mathrm{BSA}, 5 \mathrm{mg} \mathrm{mL}^{-1} \mathrm{PVP}-40$, and $0.25 \%$ v/v IGEPAL CA-630), for at least $2 \mathrm{~min}$, and then the swab was discarded. Individual or pooled samples were used immediately.

To test the compatibility of phage LFA with clinical samples, we initially used frozen, de-identified post-diagnostic molecular testing, nasopharyngeal swab extracts, a generous gift from University of Texas Medical Branch at Galveston (Dr. Scott Weaver and Ms. Nehad Saad) and Community Labs, LLC (Dr. Scott Jones). We tested individual and pooled samples. Aliquots were stored at $-80^{\circ} \mathrm{C}$ until thawed for a single experimental use and then discarded.

Then frozen, de-identified, anterior nasal swabs extracted in saline (leftovers from diagnostic molecular testing with known Ct values) were purchased from Labcorp. These deidentified clinical specimens were not considered human subjects research. Handling and testing of clinical specimens was performed under BSL-2 containment inside a Nuaire Class II Biological Safety Cabinet (UH MUA \#008-20).

\subsection{LFA phage reporters}

IgG-horseradish peroxidase (HRP)-conjugates ${ }^{39}$ (Peroxidase AffiniPure Goat Anti-Mouse IgG, Jackson ImmunoResearch, 115-035-003) were treated with periodate to oxidize the oligosaccharide residues of $\mathrm{IgG} \mathrm{Fc}{ }^{40}$ and $\mathrm{HRP}^{41}$ and create amine-reactive aldehyde groups. ${ }^{42}$ These aldehydes were then conjugated to the exposed primary amines of M13mp18 phage (Guild Biosciences) proteins via reductive amination. Briefly, $0.1 \mathrm{M}$ sodium meta periodate and goat anti-mouse antibody-HRP conjugates were mixed in $100 \mathrm{mM}$ sodium acetate, $\mathrm{pH} 5.5$, to final concentrations of $1.68 \mathrm{mg} \mathrm{mL}^{-1}$ and $0.22 \mathrm{mg} \mathrm{mL}^{-1}$, respectively, and allowed to react at room temperature for $20 \mathrm{~min}$. Unreacted periodate was removed using a Zeba Spin Desalting Column 7K MWCO (Thermo Fisher Scientific), pre-equilibrated with $200 \mathrm{mM}$ sodium carbonate, $\mathrm{pH}$ 9.6. $100 \mu \mathrm{L}$ of M13 phage $\left(9 \times 10^{13}\right.$ virions $\left.\mathrm{mL}^{-1}\right)$ in $200 \mathrm{mM}$ sodium carbonate, $\mathrm{pH}$ 9.6, was added to the oxidized antibody/HRP conjugates (estimated 30 antibody/HRP conjugates offered per phage) and allowed to react for $2.5 \mathrm{~h}$ at room temperature. Sodium cyanoborohydride ( $4 \mu \mathrm{L}$ of $5 \mathrm{M}$ in $1 \mathrm{M}$ sodium hydroxide) was added to the reaction $(400 \mu \mathrm{L})$ to a final concentration of $0.05 \mathrm{M}$ to reduce the unstable Schiff bases formed by the reaction of primary amines and aldehydes to stable secondary amines, and the mixture was incubated for 30 min. Next, hydroxylamine hydrochloride (1 $\mathrm{M}$ in DI water) was added to a final concentration of $0.05 \mathrm{M}$ to quench the reaction and the resulting solution was incubated for $30 \mathrm{~min}$. Uncoupled antibodies were removed by passing the solution through a Capto Core 700 resin (Cytiva) column (2 mL) pre-equilibrated with 1X phosphate-buffered saline solution (PBS). ${ }^{43}$ Phage construct concentration was determined using UV-Vis absorbance (Thermo NanoDrop ND-1000 UV/Vis Spectrophotometer) and the formula: virions $\mathrm{mL}^{-1}=\left[\left(\mathrm{A}_{269} \mathrm{~nm}-\mathrm{A}_{320 \mathrm{~nm}}\right) \times 6 \times 10^{16}\right] /$ genome length $^{44}$ (7,249 nt for M13mp18). Phage constructs were stored in $1 \mathrm{X}$ PBS at $4^{\circ} \mathrm{C}$ until use.

Prior to functional testing in phage LFA, the apparent enzymatic activity of the phage conjugate was measured in solution against a calibration curve of unmodified HRP enzyme using the 1-Step Ultra TMB HRP substrate in a 96well microtiter plate, with absorbance at $450 \mathrm{~nm}$ measured by a TECAN Infinite M200 PRO plate reader.

\subsection{LFA assembly and antibody striping}

A $25 \mathrm{~mm}$ wide by $300 \mathrm{~mm}$ long UniSart CN140 nitrocellulose membrane (Sartorius Stedim) and a $22 \mathrm{~mm}$ ReliaFlow $^{\mathrm{TM}}$ 440 absorbent pad (Ahlstrom-Munksjö) were placed on a 300 mm long backing card (DCN, MIBA-020) with a 2 mm overlap (Figure 1). $1 \mathrm{mg} \mathrm{mL}^{-1}$ rabbit monoclonal anti-SARSCoV-2 nucleoprotein antibodies and $0.5 \mathrm{mg} \mathrm{mL}^{-1}$ goat polyclonal anti-mouse antibodies (Arista Biologicals, ABGAM0500) in $1 \mathrm{X}$ PBS were dispensed on the membrane using a Biodot XYZ3060 system (flow rate $1 \mu \mathrm{L} \mathrm{cm}{ }^{-1}$ ) to form test and control lines, respectively. The membranes were allowed to dry at $50^{\circ} \mathrm{C}$ for $1 \mathrm{~h}$ and stored in a desiccator overnight. The membranes were cut into $3 \mathrm{~mm}$ wide strips using a ZQ2000 Guillotine Cutter (Kinbio Tech) and stored desiccated at room temperature until use.

\subsection{LFA running protocol}

Based on previous experience and a brief screening, the formulation of the LFA running/extraction buffer used in this work was $1 \mathrm{X}$ PBS (pH 7.4), $10 \mathrm{mg} \mathrm{mL}^{-1}$ BSA (blocking/passivation agent), $5 \mathrm{mg} \mathrm{mL}^{-1} \mathrm{PVP}-40$ (a neutral polymer to facilitate dispersion), and $0.25 \% \mathrm{v} / \mathrm{v}$ IGEPAL CA630 (a nonionic, non-denaturing immunoassay-compatible surfactant commonly used for virus lysis).

Recombinant SARS-CoV-2 nucleoprotein (ACRO Biosystems, NUN-C5227; calculated MW 47.3 kDa; GeneBank: QHO62115.1) in extraction buffer or negative nasal swab extract $(24 \mu \mathrm{L})$ was mixed with $6 \mu \mathrm{L}$ mouse anti-nucleoprotein antibodies (final concentration of $0.4 \mu \mathrm{g} \mathrm{mL}{ }^{-1}$ antibody). Half-strip LFA was dipped into $30 \mu \mathrm{L}$ of the sample followed by $10 \mu \mathrm{L}$ of reporter phage conjugates $\left(3 \times 10^{11}\right.$ virions $\mathrm{mL}^{-1}$ ) followed by 3 washes with $10 \mu \mathrm{L}$ of the extraction buffer. Finally, $20 \mu \mathrm{L}$ of Enhanced Chemiluminescence (ECL) substrate for HRP was applied to each strip by pipetting directly over the test and control lines and the strip was 
imaged as described below.

\subsection{Imaging and analysis}

Tests were imaged both with a laboratory imager and a smartphone.

For test optimization/development, LFA strips were imaged using an Alpha Innotech FluorChem gel documentation system equipped with a CoolSNAP K4 CCD camera (no filters; exposure time: $3 \mathrm{~s}$, binning: 4; pixel size: 7.4 $\mu \mathrm{m})$, immediately after substrate application. Images were captured at $3 \mathrm{~s}$ time intervals for at least $7 \mathrm{~min}$. Intensity profiles were extracted from the images using the plot profile tool of NIH ImageJ. ${ }^{45}$ A horizontal line was drawn along the baseline in the intensity profiles and the areas under the peaks were selected and measured using the ImageJ measure tool. These values were the intensities of the control (CL) and test line (TL) (Figure S1). The TL/CL ratio reached its maximum 6 min after adding the substrate and this time was chosen for all assays imaged on the FluorChem system (Figure S2).

The applicability of the phage LFA for point-ofcare use was demonstrated using an iPhone XR fitted with a 3-D printed lens-free accessory (Figure S3; in-house designed and made available at Thingiverse; https://www.thingiverse.com/thing:5178342) to properly position the strip directly under the (more light sensitive) back camera. An in-house developed iOS app was used for image analysis. The app reads the LFA strip by taking 10 images in raw format with predefined parameters (no flash, ISO: 2500, focus: 0, exposure: $1000 \mathrm{~ms}$, pixel size: $1.4 \mu \mathrm{m}$ ). The intensity values of blue pixels (chosen because the peak intensity of ECL light emission occurs at $440 \mathrm{~nm}$ ) from the region of interest of each image were extracted and averaged across the strip width to generate ten 1-D intensity profile arrays. To further reduce the sensor noise, the ten intensity profile arrays were averaged together into one intensity profile array. The app then determined the location of the control line (CL) by identifying the pixel with maximum intensity in the top half of the LFA strip, downstream from the test line. Next, the app validated the CL by checking the maximum intensity against a preset threshold (twice the intensity profile minimum). The CL served as the reference point to locate the test line (TL), a local maximum at $400 \pm 75$ pixels away $(5 \mathrm{~mm})$. The background (BG) was determined midway between the CL and TL by finding the local minimum at $200 \pm 75$ pixels away from the CL peak. The integrated intensity values of CL, TL, and BG were calculated using the trapezoidal rule with lower and upper integrating limits of peak location \pm 25 pixels. Finally, the value of $\mathrm{TL} / \mathrm{CL}$ ratio was determined as $\mathrm{TL} / \mathrm{CL}=$ (TL-BG)/(CL-BG). In preliminary experiments, we observed that the TL/CL ratio reached its maximum value 3 min after adding the substrate and this time was used for all smartphone measurements (Figure S4). We attribute the difference in the smartphone-based analysis time (3 min as compared to $6 \mathrm{~min}$ with the FluorChem system) to the fact that the strip was tightly constrained inside a plastic LFA cassette (Figure S3) and its side walls accelerated the flow and penetration of the HRP substrate through the nitrocellulose.

\subsection{Antibody screening on LFA}

Twelve capture antibodies (rabbit monoclonal anti-SARSCoV-2 nucleoprotein antibodies) and seven detection antibodies (mouse monoclonal anti-SARS-CoV-2 nucleoprotein antibodies) (Table 1) were screened in all possible combinations directly on LFA half strips. Each pair was initially tested with a no-analyte (negative) sample and 5 ng $\mathrm{mL}^{-1}$ SARS-CoV-2 recombinant nucleoprotein (ACRO Biosystems, NUN-C5227) in LFA extraction buffer using anti-mouse IgG/HRP phage reporters. Pairs were ranked based on the difference between the TL/CL ratios of positive (specific signal) and negative (non-specific signal) strips. In the second round of screening, antibody pairs were tested with a no-analyte and $1 \mathrm{ng} \mathrm{mL}^{-1}$ of nucleoprotein in extraction buffer. The third round of screening was performed using nasal swab extract spiked with serial dilutions of nucleoprotein.

Table 1. Commercial anti-SARS-CoV-2 nucleoprotein monoclonal antibodies were screened in the phage LFA. Final selected antibodies (capture: \#3 and detection: \#12) are highlighted in gray.

\begin{tabular}{|c|c|c|}
\hline Index & Vendor & Catalog \# \\
\hline 1 & Bioss Antibodies & m-bsm-41411M \\
\hline 2 & Ray Biotech & $5 \mathrm{~F} 7 \mathrm{~B} 3$ \\
\hline 3 & Ray Biotech & $1 \mathrm{G} 1-\mathrm{F} 2$ \\
\hline 4 & Ray Biotech & $1 \mathrm{~A} 4 \mathrm{~g} 1 \mathrm{G} 12 \mathrm{G} 12$ \\
\hline 5 & Pro Sci & 35720 \\
\hline 6 & Sino Biological & $40143-\mathrm{MM} 08$ \\
\hline 7 & Sino Biological & $40143-\mathrm{MM} 05$ \\
\hline 8 & ExonBio & NP11A7 \\
\hline 9 & ExonBio & NP5B1 \\
\hline 10 & ExonBio & NP5E2 \\
\hline 11 & ExonBio & NP11H9 \\
\hline 12 & ExonBio & $12 \mathrm{~F} 1$ \\
\hline 13 & ExonBio & NP12C1 \\
\hline 14 & ExonBio & NP12E6 \\
\hline 15 & ExonBio & NP12B8 \\
\hline 16 & Ray Biotech & $130-10760$ \\
\hline 17 & Pro Sci & $10-352$ \\
\hline 18 & Sino Biological & $40143-\mathrm{R} 004$ \\
\hline 19 & ExonBio & NP12A1 \\
\hline
\end{tabular}

\section{$2.8 \quad$ RT-qPCR testing}

We adapted the Yale SalivaDirect RT-PCR assay ${ }^{46}$ for use with nasal swab extracts. Nasal extract $(50 \mu \mathrm{L})$ was first treated with proteinase $\mathrm{K}\left(6.3 \mu \mathrm{L}\right.$ of $\left.20 \mathrm{mg} \mathrm{mL}^{-1}\right)$ by vortexing for $1 \mathrm{~min}$ (Vortex Genie 2, analog control knob at 7) followed by heat inactivation $\left(95^{\circ} \mathrm{C}, 5 \mathrm{~min}\right)$ and $5 \mu \mathrm{L}$ used as input in singleplex RT-qPCR reactions using the CDC 2019-nCoV-2 RUO pre-mixed primer and probe sets (IDT DNA Technologies; N1 and RP) and the Luna Universal Probe One-Step RT-qPCR Kit (E3006S; New England Biolabs) on an MX3005P qPCR instrument (Agilent). For each singleplex RT-qPCR reaction, $5 \mu \mathrm{L}$ of processed sample was mixed with $1.5 \mu \mathrm{L}$ of primer/probe mixture (final primer and FAM/BHQ-1-probe concentrations were $500 \mathrm{nM}$ and $125 \mathrm{nM}$, respectively), $1 \mu \mathrm{L}$ of $10 \mathrm{X}$ Luna WarmStart ${ }^{\circledR} \mathrm{RT}$ Enzyme Mix, $10 \mu \mathrm{L}$ of $2 \mathrm{X}$ Luna Universal Probe One-Step Reaction Mix, and $2.5 \mu \mathrm{L}$ of nuclease-free water. The RTPCR conditions were $10 \mathrm{~min}$ at $52^{\circ} \mathrm{C}$ (Reverse Transcription/cDNA synthesis step), 2 min at $95^{\circ} \mathrm{C}$, and 45 cycles 
of $10 \mathrm{~s}$ at $95^{\circ} \mathrm{C}$ and $30 \mathrm{~s}$ at $55^{\circ} \mathrm{C}$. Dilutions of SARS-CoV2 (2019-nCoV) RUO plasmid control containing the $\mathrm{nCoV}$ nucleoprotein gene (GenBank NC045512.2; IDT DNA Technologies; $5 \times 10^{5}$ to 50 copies per reaction) in nuclease-free water were used to construct standard curves for every run (typical observed amplification efficiency of 0.91). To confirm the input of human RNA the Hs_RPP30 plasmid control (IDT DNA) was used, which contained a portion of the single copy, human Ribunuclease P Protein subunit p30 gene. No template control reactions typically gave "not detected" (n.d.) Ct values. qPCR Control SARS-CoV-2 RNA (BEI NR-52347) was used as a positive control (1250 genome equivalents per $5 \mu \mathrm{L}$, Ct value $26.6 \pm 0.7, n=4)$.

\subsection{Clinical sensitivity with banked samples}

Initially, three banked positive clinical samples (Ct values $14.2,15.6$, and 13.2) were pooled and the pool serially diluted in fresh pooled negative nasal swab extract. The pool serial dilutions were tested in parallel by RT-qPCR and phage LFA as described in $\S 2.8$ and $\S 2.5$, respectively.

Banked, de-identified, anterior nasal swab liquid samples including 12 negative and 15 positive samples (knownCt leftovers from diagnostic molecular testing) purchased from Labcorp were tested in phage LFA. To condition the sample while minimizing analyte dilution, clinical samples were mixed $4: 1$ with a $5 \mathrm{X}$ concentrated extraction buffer to achieve a final $1 \mathrm{X}$ LFA extraction buffer and $4 \mu \mathrm{g} \mathrm{mL}{ }^{-1}$ detection antibodies. $30 \mu \mathrm{L}$ of this sample was run on an LFA strip followed by anti-mouse IgG/HRP phage reporters and the rest of the protocol was as described in $\S 2.5$.

IBM SPSS statistics software 28.0.1.0 (IBM, Armonk, NY) was used to run Mann-Whitney U tests and the receiver operating characteristic (ROC) curve analyses.

\section{Results and Discussion}

\subsection{Universal antibody-enzyme phage LFA reporters}

We chemically functionalized M13 phage proteins with antimouse IgG/HRP conjugates by oxidation of polysaccharides on antibodies and HRP enzymes to make universal, easilycustomizable ultrasensitive LFA reporters. The sample was mixed with mouse monoclonal anti-nucleoprotein detection antibodies and detection antibody-analyte complexes were captured on the test line bearing rabbit monoclonal antinucleoprotein antibodies. Anti-mouse antibody/HRP phage reporters were then added, and HRP captured on the test line catalyzed the oxidation of the chemiluminescent substrate, which generated a light signal readily detectable by an unmodified smartphone (Figure 1).

We initially investigated different strategies for the direct conjugation of anti-nucleoprotein antibodies and HRP enzymes on the primary amines of the phage coat proteins, ${ }^{47}$ including the commonly-used carbodiimide-mediated crosslinking with EDC and sulfo-NHS, Traut's reagent thiolation of primary amines for coupling to maleimide-activated antibodies, ${ }^{33,34}$ and periodate-mediated oxidation of glycosylated antibody $\mathrm{Fc}$ and HRP sugars to amine-reactive

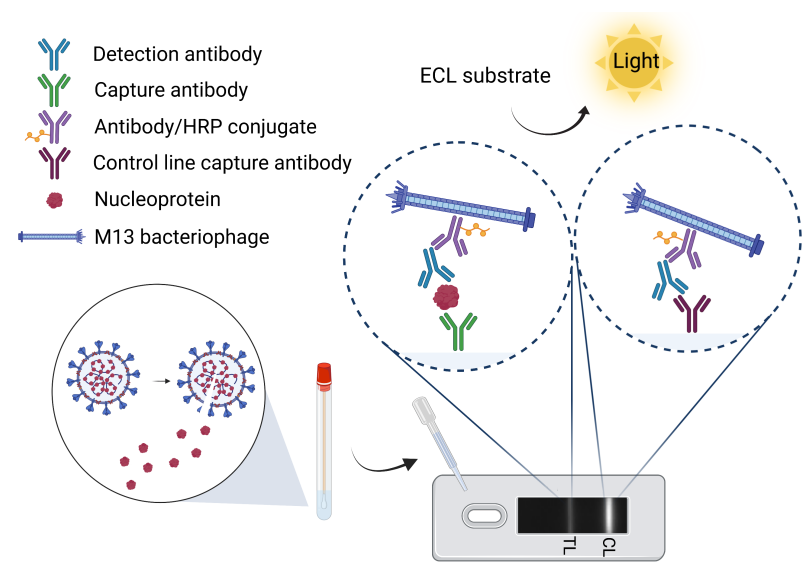

Figure 1. Schematic illustration of the chemiluminescent phage lateral flow assay for the detection of SARS-CoV-2 nucleoprotein. Nucleoprotein is sandwiched between mouse detection antibodies and rabbit capture antibodies on the LFA test line (TL) and then detected by anti-mouse antibody-HRP phage reporters. Light signal from the HRP-mediated chemiluminescent reaction is captured and analysed by a smartphone and associated app. Created with BioRender.com

aldehydes. All antibody-HRP phage conjugates tested performed well in ELISA and were captured on LFA control lines but the signal for nucleoprotein on test line was not detectable. Phage functionalized with commercial (pre-conjugated) anti-mouse IgG/HRP conjugates combined with soluble mouse anti-nucleoprotein antibodies, however, showed superior LFA analytical sensitivity.

\subsection{Antibody screening}

The performance of immunoassays depends critically upon the pair of antibodies used. ${ }^{48}$ Traditional equilibrium-based antibody screening methods such as ELISA, however, are not predictive of the behavior of antibodies when integrated in LFA format. ${ }^{5,49}$ Thus, we performed three rounds of antibody screening directly in phage LFA. First, we screened all 84 monoclonal antibody pairs against SARS-CoV-2 nucleoprotein (Table 1 ) in phage LFA using a negative (0 $\mathrm{ng} \mathrm{mL}^{-1}$ ) and a high positive (5 $\mathrm{ng} \mathrm{mL}^{-1}$ nucleoprotein) sample in extraction buffer (Figure 2). We calculated the ratio of the intensities of test and control lines and ranked the pairs based on the difference between the ratio of test line to control line of positive strips and negative strips $(\mathrm{TL} / \mathrm{CL})_{\text {positive }}-(\mathrm{TL} / \mathrm{CL})_{\text {negative }}$ for each pair (Figure 2). Interestingly, seven antibody pairs demonstrated non-specific signal (negative strip) that was greater than the specific signal (positive strip) whereas eighteen pairs demonstrated indistinguishable non-specific and specific signals. The six antibody pairs with the highest ranking and in sufficient stock (shown in bold in Figure 2) were then tested with $1 \mathrm{ng} \mathrm{mL}{ }^{-1}$ nucleoprotein in LFA extraction buffer. The differences between the TL/CL values of positive strips and negative strips $\left((\mathrm{TL} / \mathrm{CL})_{\text {positive }}-(\mathrm{TL} / \mathrm{CL})_{\text {negative }}\right)$ for the six pairs were: $8-3: 0.22,9-3: 0.08,10-3: 0.11,12-3: 0.34$, 13-3: $0.32,14-3$ : 0.34 . The two antibody pairs with the highest ranking and in sufficient stock, 13-3 and 12-3, were then tested with nucleoprotein $\left(0,0.05,0.1\right.$, and $\left.0.5 \mathrm{ng} \mathrm{mL} \mathrm{m}^{-1}\right)$ spiked in nasal swab extract. The difference in TL/CL of negative strips and low positive strips $\left(0.05 \mathrm{ng} \mathrm{mL}^{-1}\right)$ was higher for the 12-3 pair (Figure S5). Consequently, we used 
this pair, \#12, rabbit monoclonal antibody 12F1 (ExonBio) and \#3, mouse monoclonal antibody 1G1-F2 (Ray Biotech), for further test development and validation.

\begin{tabular}{|c|c|c|c|c|c|c|c|}
\hline \#8 & 0.51 & 0.42 & 0.83 & 0.30 & 0.22 & 0.01 & 0.36 \\
\hline$\# 9$ & 0.00 & 0.21 & 0.61 & 0.14 & 0.13 & 0.20 & 0.28 \\
\hline \#10 & 0.20 & 0.22 & 0.75 & 0.16 & 0.18 & 0.28 & 0.36 \\
\hline \#11 & 0.17 & 0.21 & 0.67 & 0.14 & 0.03 & -0.03 & 0.08 \\
\hline 중 \#12 & 0.37 & 0.61 & 0.96 & 0.28 & 0.02 & 0.02 & 0.36 \\
\hline 宔 $\# 13$ & 0.37 & 0.60 & 0.91 & 0.26 & -0.03 & 0.04 & 0.28 \\
\hline$\stackrel{\varrho}{\frac{\varrho}{3}} \# 14$ & 0.31 & 0.59 & 0.83 & 0.26 & 0.04 & 0.01 & 0.36 \\
\hline ర \#15 & 0.19 & -0.01 & 0.08 & 0.02 & 0.16 & 0.28 & 0.08 \\
\hline \#16 & 0.01 & 0.04 & 0.01 & 0.00 & 0.02 & -0.02 & 0.21 \\
\hline \#17 & 0.11 & 0.54 & 0.45 & 0.14 & 0.12 & 0.27 & 0.22 \\
\hline \#18 & 0.02 & -0.07 & 0.15 & -0.01 & 0.03 & 0.02 & 0.22 \\
\hline \#19 & 0.24 & 0.85 & 0.74 & 0.34 & -0.01 & 0.01 & 0.27 \\
\hline & \# & \#2 & \#3 & \#4 & \# & \#6 & \#7 \\
\hline
\end{tabular}

Figure 2. Phage LFA-based antibody screening. The performance of the 84 antibody pairs was initially evaluated with a no-target and $5 \mathrm{ng} \mathrm{mL}^{-1}$ nucleoprotein sample in LFA extraction buffer. Values shown are the differences between the TL/CL ratios of positive and negative strips; darker blues correspond to higher differences. The top 6 antibody pairs that were available in sufficient stock (shown in bold borders) were chosen for the next round of screening using $1 \mathrm{ng} \mathrm{mL} \mathrm{mL}^{-1}$ nucleoprotein.

\subsection{Analytical sensitivity with contrived samples}

Following antibody screening we evaluated the analytical sensitivity of the chosen antibody pair in phage LFA with nasal swab extracts (presumed negative for COVID-19) spiked with recombinant nucleoprotein $\left(25 \mathrm{pg} \mathrm{mL}^{-1}\right.$ to 500 pg $\mathrm{mL}^{-1}$; Figure 3$)$. The signal increased linearly with increasing nucleoprotein concentration and the limit of detection (LoD), defined as the lowest analyte concentration for which the signal is above the Average blank $+3 \times \mathrm{STD}_{\text {blank }}$, was $25 \mathrm{pg} \mathrm{mL} \mathrm{m}^{-1}(0.53 \mathrm{pM})$. There is no general consensus on the required analytical sensitivity to ensure clinical utility. A recent study ${ }^{50}$ using the Quanterix SIMOA technology showed a median nucleoprotein concentration of 215 pg $\mathrm{mL}^{-1}$ in PCR-positive banked swabs $(n=148$ with 71 samples with $\geq 1000 \mathrm{pg} \mathrm{mL}^{-1}$ ). Thus the sub-picomolar LoD of the phage LFA in nasal swab extract is promising and better than the LoD in recently reported SARS-CoV-2 nucleoprotein colorimetric LFAs, as discussed in $§ 3.5$.

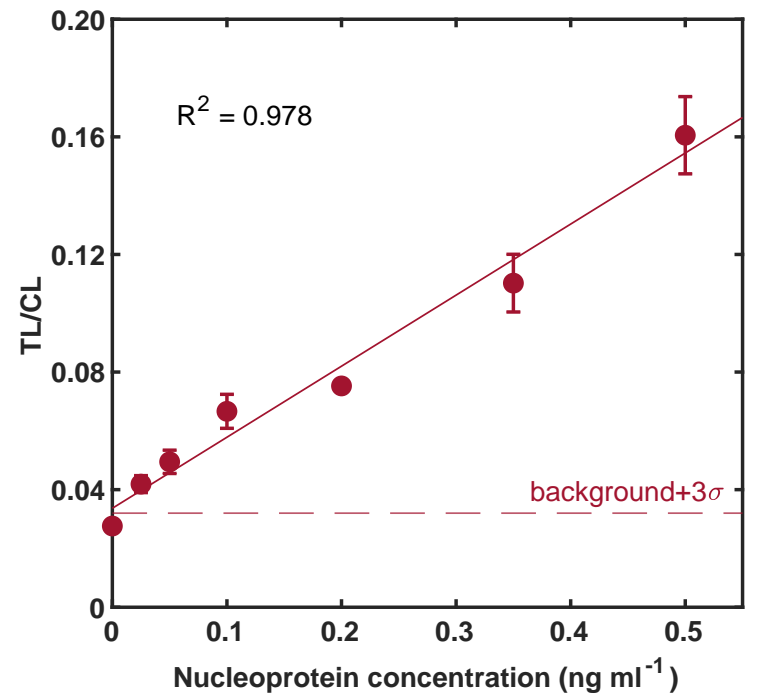

Figure 3. Sub-picomolar detection of nucleoprotein using the phage LFA. Nucleoprotein was spiked in presumed-negative nasal swab extracts; capture: rabbit monoclonal antibody $12 \mathrm{~F} 1$ (\#12); ExonBio and detection: mouse monoclonal antibody 1G1-F2 (\#3); Ray Biotech. LFA strips were imaged on the FluorChem gel documentation system. Data are mean \pm s.d.; $n=4$. The dashed red line denotes the estimated background as the average plus three times the standard deviation $(\mu+3 \sigma)$ of the negative tests.

\subsection{Clinical sensitivity with nasal swab extracts}

To assess the clinical utility of phage LFA, three COVID-19 positive swab extracts were pooled together, serially diluted in negative nasal swab extract, and tested in the RT-PCR assay and in phage LFA.

Here, we adapted the Yale SalivaDirect assay that circumvents RNA extraction and performed RT-PCR directly on nasal swab extracts. We obtained $\mathrm{Ct}$ values for Labcorp positive samples (received frozen and then thawed) that were an average $1.9( \pm 2.3)$ cycles higher than the Labcorp-supplied $\mathrm{Ct}$ values (with a Spearman's correlation coefficient of 0.89 ) demonstrating that our direct RT-PCR was sufficient for the needs of our study (to benchmark serially-diluted pooled samples prior to LFA testing). All Labcorp negative samples had Ct values greater than 40 .

The Ct values determined for the 10, 50, 100, and 1,000fold diluted pools were 21.6, 23.4, 25.6, and 29.4, respectively. The LFA signals for all diluted pools tested, including the pool with $\mathrm{Ct}$ value of 28 , were higher than the signal for the negative sample (Figure S6). These results underscore the high sensitivity of the phage LFA. Recent studies have tried to correlate the performance of LFA with PCR Ct values $^{51-53}$ but differently-calibrated PCR systems, different workflows, and fundamentally different targets (nucleic acid vs. protein) hinder the drawing of general conclusions.

We then tested 27 de-identified nasal swab extracts from Labcorp, including 15 positive samples with known Ct values ranging from 18.7 to 29.6 (3 samples with $\mathrm{Ct}$ value greater than 28) and 12 negative samples (Ct values not reported but greater than 40) (Table S1). The LFA signals for PCRpositive samples were statistically different from the negative samples (Mann-Whitney U test, $p<0.001$; Figure 4; Figure S7: LFA signal and Ct value for all positive samples tested) 
indicating that phage LFA was sufficiently sensitive to discriminate between positive and negative samples. Based on the ROC analysis (Figure S8), we achieved a maximum sensitivity of $93.3 \%$ at $100 \%$ specificity (area under the ROC curve $($ AUC $)=0.994 ; 95 \%$ CI 0.977-1.00).

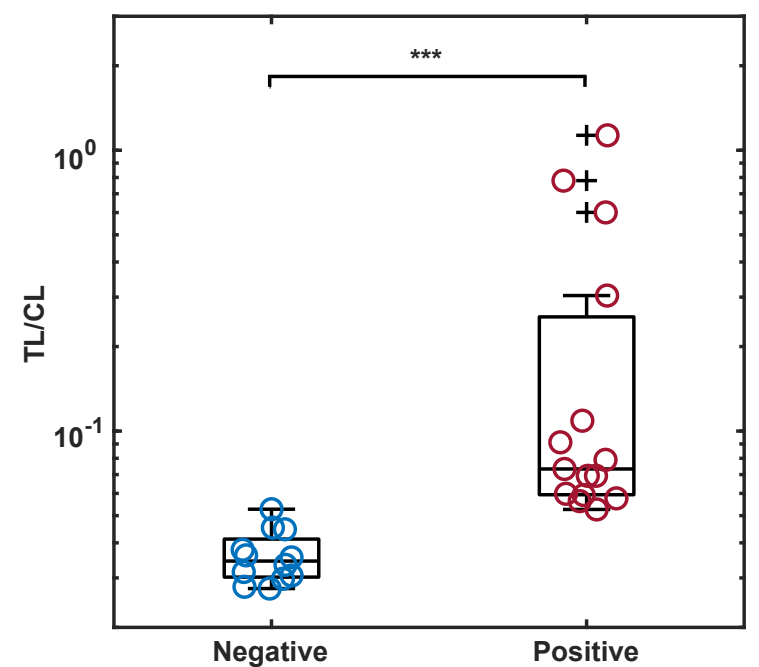

Figure 4. Individual results of clinical specimens (nasal swab extracts from Labcorp) tested using the phage LFA and read with lab instrumentation. The box plots display the phage LFA TL/CL ratios of negative $(n=12)$ and positive samples $(n=15)$. Horizontal lines on each box plot, from bottom to top beginning with the bottom whisker are: 10th percentile, 25th percentile, median, 75th percentile, and 90th percentile. + symbols indicate outliers (low Ct value samples, 21.1, 19.5 and 18.7). *** symbol indicates that the two groups are statistically different by the Mann-Whitney $\mathrm{U}$ test, $p<0.001$. TL/CL as a function of $\mathrm{Ct}$ value is shown in Figure S7.

\subsection{Smartphone-based LFA}

Smartphones provide a now-ubiquitous, portable, and userfriendly platform to image and interpret optically-reporting LFAs. ${ }^{54}$ Moreover, the wavelength of the ECL chemiluminescent emission matches the smartphone camera spectral sensitivity curve. ${ }^{55,56}$ We developed a 3D-printed attachment that positions the LFA strip directly under the back camera of the iPhone XR and blocks out all ambient light, maintaining a dark environment (Figure S3). An iOS image analysis app was developed in-house (a typical analysis screenshot is shown in Figure S3).

We tested nucleoprotein serially diluted in nasal swab extract and read the LFA strips using the iPhone reader. The TL/CL ratio increased with increasing nucleoprotein concentration (Figure 5) and the LoD was estimated at $100 \mathrm{pg}$ $\mathrm{mL}^{-1}$. The smartphone-based LoD value was higher than the LoD estimated using the CCD camera of the bulkier and more expensive FluorChem imaging system. Nevertheless, the off-the-shelf, portable and affordable smartphone reader showed a low and clinically-relevant ${ }^{50}$ LoD in addition to the user-friendly and point-of-care features of the smartphone platform.

The LoD of the phage LFA is better than most recently reported values (for the detection of SARS-CoV-2 nucleoprotein or spike protein) in equipment-free colorimet- ric LFAs (gold nanoparticles, ${ }^{3,7,8}$ latex particles, ${ }^{6}$ cellulose nanobeads; ${ }^{12,13}$ Table S2). The analytical sensitivity of phage LFA is even comparable to that of fluorescent LFAs read by costly and specialized readers. ${ }^{10,11}$ Post-LFA signal amplification was shown to greatly improve the LoD of gold LFAs but at the expense of increased background that may hinder reliable visual interpretation ${ }^{9}$ or economy and $\mathrm{POC}$ applicability, since the enhanced LFA strip is read with a complex reader assembly. ${ }^{57}$

Next, we tested 26 nasal swab clinical samples including 15 positive and 11 negative samples, in phage LFA and read and analyzed the signals using the smartphone (Figure 6; Figure S9: LFA signal and Ct value for all positive samples tested). Using a Mann-Whitney U test, negative samples tested and positive samples tested were confirmed to be statistically different $(p<0.001)$ indicating that the smartphone-read phage LFA was sufficiently sensitive to discriminate between positive and negative clinical samples. Based on the ROC analysis (Figure S10), we achieved a maximum sensitivity of $80 \%$ at $100 \%$ specificity (area under the ROC curve (AUC) $=0.939 ; 95 \%$ CI $0.854-1.00)$.

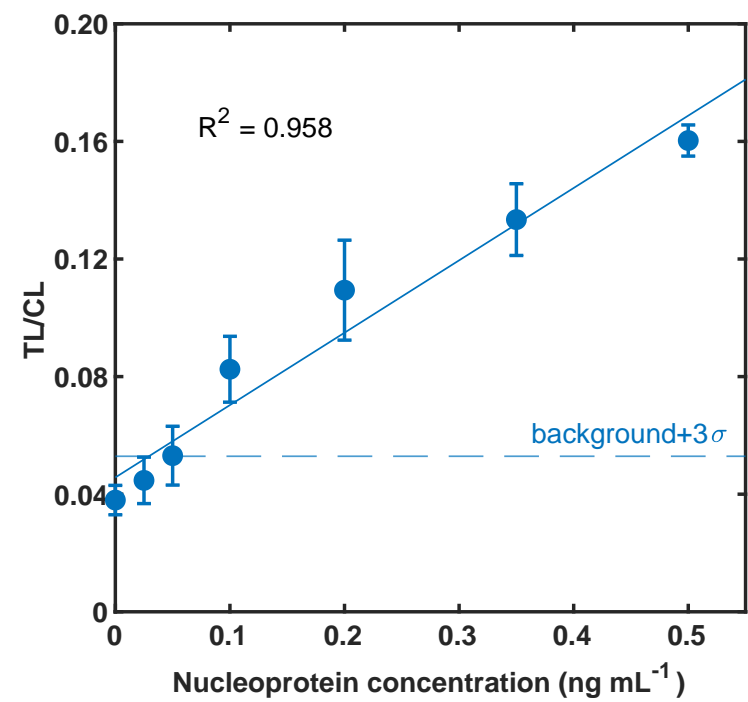

Figure 5. Smartphone-based detection of nucleoprotein using the phage LFA. Nucleoprotein was spiked in presumed negative nasal swab extracts; capture: rabbit monoclonal antibody $12 \mathrm{~F} 1$ (\#12); ExonBio and detection: mouse monoclonal antibody 1G1F2 (\#3); Ray Biotech. Data are mean \pm s.d.; $n=3$. The dashed blue line denotes the estimated background as the average plus three times the standard deviation $(\mu+3 \sigma)$ of the negative tests.

\section{Conclusions}

We engineered universal LFA reporters by conjugating antimouse IgG/HRP conjugates to M13 phage and demonstrated a translation-ready, ultrasensitive phage LFA for the point-of-care detection of SARS-CoV-2 nucleoprotein in clinical samples. By combining the advantages of phage reporters and enzyme-generated chemiluminescence, we enhanced the sensitivity of LFAs and demonstrated an LoD of $100 \mathrm{pg} \mathrm{mL}{ }^{-1}$ for SARS-CoV-2 nucleoprotein spiked in nasal extract and read by an off-the-shelf smartphone. Furthermore, the phage LFA demonstrated excellent clinical sensitivity with 15 banked PCR-confirmed positive nasal swab extracts of $\mathrm{Ct}$ values between 18.7 to 29.6. Further im- 


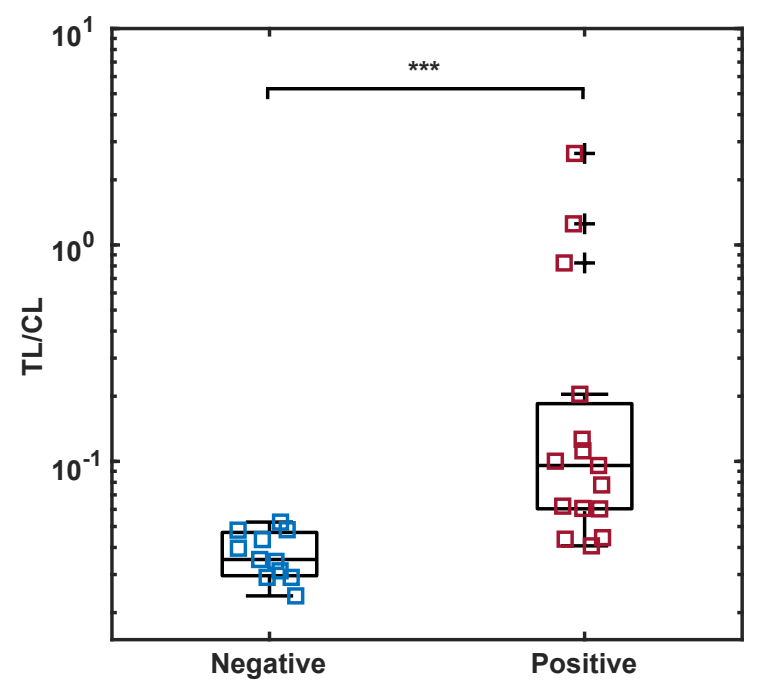

Figure 6. Individual results of clinical specimens (nasal swab extracts from Labcorp) tested using the phage LFA and read with a smartphone. The box plots display the LFA signals (TL/CL) of negative $(n=11)$ and positive samples $(n=15)$. Horizontal lines on each box plot, from bottom to top beginning with the bottom whisker are: 10th percentile, 25th percentile, median, 75th percentile, and 90th percentile. + symbols indicate outliers (low Ct value samples, 21.1, 19.5 and 18.7). *** symbol indicates that the two groups are statistically different by the Mann-Whitney $\mathrm{U}$ test, $p<0.001$. TL/CL as a function of $\mathrm{Ct}$ value is shown in Figure S9.

provements in the smartphone reader, e.g. by the addition of a macro lens for light focusing, could enhance detection sensitivity. Moreover, given recent advances in device ${ }^{58-61}$ and materials ${ }^{62,63}$ engineering facilitating the adoption of chemiluminescence into point-of-care diagnostics, our phage LFA reporters would enable lateral flow tests that are rapid, ultrasensitive, user-friendly, equipment-free and potentially rapidly widely-deployable, thereby enabling large-scale diagnostic testing.

\section{Acknowledgements}

We gratefully acknowledge Dr. Scott Weaver and Ms. Nehad Saad at University of Texas Medical Branch at Galveston and Dr. Scott Jones at Community Labs, LLC (San Antonio, TX) for the de-identified positive and negative swab extracts. We thank Krisha Doshi at ExonBio and Lawrence Gray at IDT for helpful discussions. The following reagent was deposited by the Centers for Disease Control and Prevention and obtained through BEI Resources, NIAID, NIH: Quantitative PCR (qPCR) Control RNA from Heat-Inactivated SARS-Related Coronavirus 2, Isolate USA-WA1/2020, NR-52347. We gratefully acknowledge financial support from the University of Houston "Grants to Enhance Research on COVID-19 and the Pandemic" Program (Grant \#000180936 and Grant \#00180943), the NIH Rapid Acceleration of Diagnostics (RADx) program, NIH/NIAMS (Grant \#1R01AR072742-01), DOD CDMRP (Grant \#W81XWH-21-1-0975), the National Science Foundation (CBET-1803728 and CBET-1928334), and the Welch Foundation (E-1869).

\section{References}

(1) Mak, G. C. K.; Cheng, P. K. C.; Lau, S. S. Y.; Wong, K. K. Y. Lau, C. S.; Lam, E. T. K.; Chan, R. C. W.; Tsang, D. N. C. Evaluation of rapid antigen test for detection of SARS-CoV-2 virus. J. Clin. Virol. 2020, 219, 104500.

(2) Larremore, D. B.; Wilder, B.; Lester, E.; Shehata, S. Burke, J. M.; Hay, J. A.; Tambe, M.; Mina, M. J.; Parker, R. Test sensitivity is secondary to frequency and turnaround time for COVID-19 screening. Sci. Adv, 2021, 7(1), eabd5393.

(3) Baker, A. N. et al. The SARS-COV-2 Spike Protein Binds Sialic Acids and Enables Rapid Detection in a Lateral Flow Point of Care Diagnostic Device. ACS Cent. Sci. 2020, 6(11), 20462052

(4) Yan, Y.; Chang, L.; Wang, L. Laboratory testing of SARSCoV, MERS-CoV, and SARS-CoV-2 (2019-nCoV): Current status, challenges, and countermeasures. Rev. Med. Virol. 2020 $30(3), \mathrm{e} 2106$

(5) Cate, D. M. et al. Antibody Screening Results for AntiNucleocapsid Antibodies Toward the Development of a Lateral Flow Assay to Detect SARS-CoV-2 Nucleocapsid Protein. ACS Omega 2021, 6(39), 25116-25123.

(6) Grant, B. D.; Anderson, C. E.; Williford, J. R.; Alonzo, L. F.; Glukhova, V. A.; Boyle, D. S.; Weigl, B. H.; ; Nichols, K. P. SARS-CoV-2 Coronavirus Nucleocapsid Antigen-Detecting Half-Strip Lateral Flow Assay Toward the Development of Point of Care Tests Using Commercially Available Reagents. Anal. Chem. 2020, 92(16), 11305-11309.

(7) Mertens, P. et al. Development and Potential Usefulness of the COVID-19 Ag Respi-Strip Diagnostic Assay in a Pandemic Context. Front. Med. 2020, 7, 225.

(8) Liu, D.; Wu, F.; Cen, Y.; Ye, L.; Shi, X.; Huang, Y.; Fang, S.; $\mathrm{Ma}, \mathrm{L}$. Comparative research on nucleocapsid and spike glycoprotein as the rapid immunodetection targets of COVID-19 and establishment of immunoassay strips. Mol. Immunol. 2021 131, 6-12.

(9) Peng, T.; Jiao, X.; Liang, Z.; Zhao, H.; Zhao, Y.; Xie, J.; Jiang, Y.; Yu, X.; Fang, X.; Dai, X. Lateral Flow Immunoassay Coupled with Copper Enhancement for Rapid and Sensitive SARS-CoV-2 Nucleocapsid Protein Detection. Biosensors 2022, 12(1), 13

(10) Wang, C.; Cheng, X.; Liu, L.; Zhang, X.; Yang, X.; Zheng, S.; Rong, Z.; ; Wang, S. Ultrasensitive and Simultaneous Detection of Two Specific SARS- CoV-2 Antigens in Human Specimens Using Direct/Enrichment Dual-Mode Fluorescence Lateral Flow Immunoassay. ACS Appl. Mater. Interfaces 2021 13(34), 40342-40353.

(11) Wang, C.; Yang, X.; Zheng, S.; Cheng, X.; Xiao, R.; Li, Q. Wang, W.; Liu, X.; Wang, S. Development of an ultrasensitive fluorescent immunochromatographic assay based on multilayer quantum dot nanobead for simultaneous detection of SARSCoV-2 antigen and influenza A virus. Sens. Actuators $B$ Chem. 2021, 345, 130372 .

(12) Kim, H.-Y.; Lee, J.-H.; Kim, M. J.; Park, S. C.; Choi, M.; Lee, W.; Ku, K. B.; Kim, B. T.; Park, E. C.; Kim, H. G.; Kim, S. I. Development of a SARS-CoV-2-specific biosensor for antigen detection using scFv-Fc fusion proteins. Biosens. Bioelectron. 2020, 175, 112868.

(13) Lee, J.-H.; Choi, M.; Jung, Y.; Lee, S. K.; Lee, C.-S.; Kim, J. Kim, J.; Kim, N. H.; Kim, B.-T.; Kim, H. G. A novel rapid detection for SARS-CoV-2 spike 1 antigens using human angiotensin converting enzyme 2 (ACE2). Biosens. Bioelectron. 2021, 171, 112715.

(14) Mahmoudinobar, F.; Britton, D.; Montclare, J. K. Proteinbased lateral flow assays for COVID-19 detection. Protein Eng. Des. Sel. 2021, 34, gzab010.

(15) In Vitro Diagnostics EUAs - Antigen Diagnostic Tests for SARSCoV-2. https://www.fda.gov/medical-devices/coronavirus-di sease-2019-covid-19-emergency-use-authorizations-medical-d evices/in-vitro-diagnostics-euas-antigen-diagnostic-testssars-cov-2, (accessed: 12.12.2021).

(16) Guidance for Clinicians on the Use of Rapid Influenza Diagnostic Tests. https://www.cdc.gov/flu/pdf/professionals/diagnosi s/clinician_guidance_ridt.pdf, (accessed: 12.12.2021).

(17) Respiratory Syncytial Virus Infection (RSV). https://www.cdc. gov/rsv/clinical/index.html, (accessed: 12.12.2021).

(18) Koller, G.; Morrell, A. P.; Galão, R. P.; Pickering, S.; MacMahon, E.; Johnson, J.; Ignatyev, K.; Neil, S. J. D.; Elsharkawy, S.; Fleck, R.; Machado, P. M. P.; Addison, O. More than the Eye Can See: Shedding New Light on SARS-CoV-2 Lateral Flow Device-Based Immunoassays. ACS Appl. Mater. Interfaces 2021, 13(22), 25694-25700.

(19) Bishop, J. D.; Hsieh, H. V.; Gasperino, D. J.; Weigl, B. H. Sensitivity enhancement in lateral flow assays: a systems perspective. Lab Chip 2019, 19(15), 2486-2499.

(20) Gong, X.; Cai, J.; Zhang, B.; Zhao, Q.; Piao, J.; Peng, W. Gao, W.; Zhou, D.; Zhao, M.; Chang, J. A review of fluorescent signal-based lateral flow immunochromatographic strips. J. Mater. Chem. B 2017, 5, 5079-5091.

(21) Liang, R.-L.; Xu, X.-P.; Liu, T.-C.; Zhou, J.-W.; Wang, X.G.; Ren, Z.-Q.; Hao, F.; Wu, Y.-S. Rapid and sensitive lateral flow immunoassay method for determining alpha fetoprotein in 
serum using europium (III) chelate microparticles-based lateral flow test strips. Anal. Chim. Acta 2015, 891, 277-283.

(22) Lai, X.-H.; Liang, R.-L.; Liu, T.-C.; Dong, Z.-N.; Wu, Y.-S.; Li, L.-H. A Fluorescence Immunochromatographic Assay Using Europium (III) Chelate Microparticles for Rapid, Quantitative and Sensitive Detection of Creatine Kinase MB. J. Fluoresc. 2016, 26, 987-996.

(23) Li, Z.; Wang, Y.; Wang, J.; Tang, Z.; Pounds, J. G.; Lin, Y. Rapid and Sensitive Detection of Protein Biomarker Using a Portable Fluorescence Biosensor Based on Quantum Dots and a Lateral Flow Test Strip. Anal. Chem. 2010, 82(16), 7008-7014

(24) Shah, K. G.; Singh, V.; Kauffman, P. C.; Abe, K.; Yager, P. Mobile Phone Ratiometric Imaging Enables Highly Sensitive Fluorescence Lateral Flow Immunoassays without External Optical Filters. Anal. Chem. 2018, 90(11), 6967-6974.

(25) Rong, Z.; Wang, Q.; Sun, N.; Jia, X.; Wang, K.; Xiao, R.; Wang, S. Smartphone-based fluorescent lateral flow immunoassay platform for highly sensitive point-of-care detection of $Z i k a$ virus nonstructural protein 1. Anal. Chim. Acta 2019, 1055, $140-147$

(26) Zou, R.; Chang, Y.; Zhang, T.; Si, F.; Liu, Y.; Zhao, Y.; Liu, Y.; Zhang, M.; Yu, X.; Qiao, X.; Zhu, G.; Guo, Y. UpConverting Nanoparticle-Based Immunochromatographic Strip for Multi-Residue Detection of Three Organophosphorus Pesticides in Food. Front. Chem. 2019, 7, 18.

(27) Nilghaz, A.; Mousavi, S. M.; Tian, J.; Cao, R.; Guijt, R. M.; Wang, X. Noble-Metal Nanoparticle-Based Colorimetric Diagnostic Assays for Point-of-Need Applications. ACS Appl. Nano Mater. 2021, 4(12), 12808-12824.

(28) Guteneva, N. V.; Znoyko, S. L.; Orlov, A. V.; Nikitin, M. P.; Nikitin, P. I. Rapid lateral flow assays based on the quantification of magnetic nanoparticle labels for multiplexed immunodetection of small molecules: application to the determination of drugs of abuse. Mikrochim. Acta 2019, 186, 621.

(29) Moyano, A.; Serrano-Pertierra, E.; Salvador, M.; MartínezGarcía, J. C.; Rivas, M.; Blanco-López, M. C. Magnetic Lateral Flow Immunoassays. Diagnostics 2020, 10(5), 288.

(30) Jiang, N.; Ahmed, R.; Damayantharan, M.; Ünal, B.; Butt, H.; Yetisen, A. K. Lateral and Vertical Flow Assays for Point-ofCare Diagnostics. Adv. Healthc. Mater. 2019, 8(14), 1900244

(31) Wang, Z.; Zhao, J.; Xu, X.; Guo, L.; Xu, L.; Sun, M.; Hu, S. Kuang, H.; Xu, C.; Li, A. An Overview for the NanoparticlesBased Quantitative Lateral Flow Assay. Small Methods 2021, 2101143 .

(32) Wong, R., Tse, H., Eds. Lateral Flow Immunoassay; Humana Press, 2009.

(33) Adhikari, M.; Dhamane, S.; Hagström, A. E. V.; Garvey, G.; Chen, W.-H.; Kourentzi, K.; Strych, U.; Willson, R. C. Functionalized viral nanoparticles as ultrasensitive reporters in lateral-flow assays. Analyst 2013, 138, 5584-5587.

(34) Adhikari, M.; Strych, U.; Kim, J.; Goux, H.; Dhamane, S. Poongavanam, M.-V.; Hagström, A. E. V.; Kourentzi, K.; Conrad, J. C.; Willson, R. C. Aptamer-Phage Reporters for Ultrasensitive Lateral Flow Assays. Anal. Chem. 2015, 87(23), 11660-11665.

(35) Hagström, A. E. V.; Garvey, G.; Paterson, A. S.; Dhamane, S.; Adhikari, M.; Estes, M. K.; Strych, U.; Kourentzi, K.; Atmar, R. L.; Willson, R. C. Sensitive Detection of Norovirus Using Phage Nanoparticle Reporters in Lateral-Flow Assay. PLoS One 2015, $10(5)$, e0126571.

(36) Kim, J.; Adhikari, M.; Dhamane, S.; Hagström, A. E. V.; Kourentzi, K.; Strych, U.; Willson, R. C.; ; Conrad, J. C. Detection of Viruses By Counting Single Fluorescent Genetically Biotinylated Reporter Immunophage Using a Lateral Flow Assay. ACS Appl. Mater. Interfaces 2015, 7(4), 2891-2898.

(37) Kim, J.; Poling-Skutvik, R.; Trabuco, J. R. C.; Kourentzi, K.; Willsona, R. C.; ; Conrad, J. C. Orientational binding modes of reporters in a viral-nanoparticle lateral flow assay. Analyst 2017, 142, 55-64.

(38) Kim, J.; Vu, B.; Kourentzi, K.; Willson, R. C.; Conrad, J. C. Increasing Binding Efficiency via Reporter Shape and Flux in a Viral Nanoparticle Lateral-Flow Assay. ACS Appl. Mater. Interfaces 2017, 9(8), 6878-6884.

(39) Nakane, P. K.; Kawaoi, A. Peroxidase-labeled antibody. A new method of conjugation. J. Histochem. Cytochem. 1974, 22(12) 1084-1091.

(40) Raju, T. S.; Briggs, J. B.; Borge, S. M.; Jones, A. J. S Species-specific variation in glycosylation of IgG: evidence for the species-specific sialylation and branch-specific galactosylation and importance for engineering recombinant glycoprotein therapeutics. Glycobiology 2000, 10(5), 477-486.

(41) Yang, B. Y.; Gray, J. S. S.; Montgomery, R. The glycans of horseradish peroxidase. Carbohydr. Res. 1996, 287(2), 203212 .

(42) Hermanson, G. T. Bioconjugate Techniques, 2nd ed.; Elsevier, 2008.

(43) Dhamane, S.; Patil, U. Smith, M.; Adhikari, M.; Nazem, A.; Conrad, J. C.; Kourentzi, K.; Willson, R. C. Isocratic reporterexclusion immunoassay using restricted-access adsorbents. Analyst 2021, 146(15), 4835-4840.

(44) Velappan, N.; Fisher, H. E.; Pesavento, E.; Chasteen, L.; D'Angelo, S.; Kiss, C.; Longmire, M.; Pavlik, P.; Bradbury, A. R. M. A comprehensive analysis of filamentous phage display vectors for cytoplasmic proteins: an analysis with different fluorescent proteins. Nucleic Acids Res. 2010, 38(4), e22.

(45) Schneider, C. A.; Rasband, W. S.; Eliceiri, K. W. NIH Image to ImageJ: 25 years of image analysis. Nat. Methods 2012,9, 671-675.

(46) Vogels, C. B. F. et al. SalivaDirect: A simplified and flexible platform to enhance SARS-CoV-2 testing capacity. Med 2021, 2(3), 263-280.e6.

(47) Li, K.; Chen, Y.; Li, S.; Nguyen, H. G.; Niu, Z.; You, S.; Mello, C. M.; Lu, X.; Wang, Q. Chemical modification of M13 bacteriophage and its application in cancer cell imaging. Bioconjugate Chem. 2010, 21(7), 1369-1377.

(48) Brooks, B. D.; Albertson, A. E.; Jones, J. A.; Speare, J. O. Lewis, R. V. Efficient screening of high-signal and lowbackground antibody pairs in the bio-bar code assay using prion protein as the target. Anal. Biochem. 2008, 382(1), 60-62.

(49) Hsieh, H. V.; Dantzler, J. L.; Weigl, B. H. Analytical Tools to Improve Optimization Procedures for Lateral Flow Assays. Diagnostics 2017, 7(2), 29.

(50) Olsen, D. A.; Brasen, C. L.; Kahns, S.; Madsen, J. B. Kierkegaard, H.; Christensen, H.; Jensen, A.; Sydenham, T. V. Møller, J. K.; Madsen, J. S.; Brandslund, I. Quantifying SARSCoV-2 nucleocapsid antigen in oropharyngeal swabs using single molecule array technology. Sci. Rep. 2021, 11, 20323.

(51) Pickering, S. et al. Comparative performance of SARS-CoV-2 lateral flow antigen tests and association with detection of infectious virus in clinical specimens: a single-centre laboratory evaluation study. Lancet Microbe 2021, 2(9), e461-e471.

(52) Mak, G. C. K.; Lau, S. S. Y.; Wong, K. K. Y.; Chow, N. L. S.; Lau, C. S.; Lam, E. T. K.; Chan, R. C. W.; Tsang, D. N. C Analytical sensitivity and clinical sensitivity of the three rapid antigen detection kits for detection of SARS-CoV-2 virus. J. Clin. Virol. 2020, 133, 104684.

(53) Petersen, I.; Crozier, A.; Buchan, I.; Mina, M. J.; Bartlett, J. W Recalibrating SARS-CoV-2 Antigen Rapid Lateral Flow Test Relative Sensitivity from Validation Studies to Absolute Sensitivity for Indicating Individuals Shedding Transmissible Virus. Clin. Epidemiol. 2021, 13, 935-940.

(54) Hernández-Neuta, I.; Neumann, F.; Brightmeyer, J.; Ba Tis, T. Madaboosi, N.; Wei, Q.; Ozcan, A.; Nilsson, M. Smartphonebased clinical diagnostics: towards democratization of evidencebased health care. J. Intern. Med. 2019, 285, 19-39.

(55) Hussain, I.; Bowden, A. K. Smartphone-based optical spectroscopic platforms for biomedical applications: a review [Invited]. Biomed. Opt. Express 2021, 12, 1974-1998.

(56) Burggraaff, O.; Schmidt, N.; Zamorano, J.; Pauly, K.; Pascual, S.; Tapia, C.; Spyrakos, E.; ; Snik, F. Standardized spectral and radiometric calibration of consumer cameras. Opt. Express 2019, 27, 19075-19101.

(57) Liu, Y.; Zhan, L.; Shen, J. W.; Baro, B.; Alemany, A.; Sackrison, J.; Mitjá, O.; Bischof, J. C. fM-aM Detection of the SARS CoV-2 Antigen by Advanced Lateral Flow Immunoassay Based on Gold Nanospheres. ACS Appl. Nano Mater. 2021, 4(12), $13826-13837$.

(58) Park, J. Y.; Kricka, L. J. Prospects for the commercialization of chemiluminescence-based point-of-care and on-site testing devices. Anal. Bioanal. Chem. 2014, 406, 5631-5637.

(59) Deng, J.; Yang, M.; Wu, J.; Zhang, W.; Jiang, X. A SelfContained Chemiluminescent Lateral Flow Assay for Point-ofCare Testing. Anal. Chem. 2018, 90(15), 9132-9137.

(60) Zhang, J.; Gui, X.; Zheng, Q.; Chen, Y.; Ge, S.; Zhang, J. $\mathrm{Xia}, \mathrm{N}$. An HRP-labeled lateral flow immunoassay for rapid simultaneous detection and differentiation of influenza $\mathrm{A}$ and $\mathrm{B}$ viruses. J. Med. Virol. 2019, 91(3), 503-507.

(61) Axelrod, T.; Eltzov, E.; Marks, R. S. Capture-Layer Lateral Flow Immunoassay: A New Platform Validated in the Detection and Quantification of Dengue NS1. ACS Omega 2020, 5(18), 10433-10440.

(62) Ghosh, S.; Ahn, C. H. Lyophilization of chemiluminescent substrate reagents for high-sensitive microchannel-based lateral flow assay (MLFA) in point-of-care (POC) diagnostic system. Analyst 2019, 144, 2109-2119.

(63) Díaz-González, M.; de la Escosura-Muñiz, A. Strip modification and alternative architectures for signal amplification in nanoparticle-based lateral flow assays. Anal. Bioanal. Chem. 2021, 413, 4111-4117. 